\title{
Intranasal Immunization of Mice with CpG DNA Induces Strong Systemic and Mucosal Responses That Are Influenced by Other Mucosal Adjuvants and Antigen Distribution
}

\author{
Michael J. McCluskie, ${ }^{1}$ Risini D. Weeratna ${ }^{1}$ and Heather L. Davis ${ }^{1,2,3,4}$ \\ ${ }^{1}$ Loeb Health Research Institute, Ottawa Hospital, Ottawa, Canada \\ ${ }^{2}$ Department of Biochemistry, Microbiology and Immunology, Faculty of Medicine, \\ ${ }^{3}$ School of Rehabilitation Sciences, Faculty of Health Sciences, University of \\ Ottawa, Ottawa, Canada \\ ${ }^{4}$ Coley Pharmaceutical Group, Inc., Wellesley, Massachusetts, U.S.A.
}

Accepted June 22, 2000.

\begin{abstract}
Background: Synthetic oligodeoxynucleotides (ODN) containing immunostimulatory cytosine-guanine phosphate-linked dinucleotide (CpG) motifs are potent systemic and mucosal adjuvants in mice that have synergistic action with numerous other adjuvants, including alum and cholera toxin (CT). Herein, we evaluate CpG ODN with intranasal (IN) delivery of purified hepatitis B surface antigen (HBsAg), relative to and in combination with CT, Escherichia coli heat labile enterotoxin (LT), the B subunit of CT (CTB), and a nontoxic derivative of LT (LTK63).

Materials and Methods: BALB/c mice were immunized by IN administration of HBsAg, alone or combined with CT, LT, CTB, or LTK63, and/or CpG ODN, or non-CpG control ODN. In addition, the effect of low-or high-volume administration was assessed, in order to target upper respiratory or entire respiratory tract, respectively. HBsAg-specific systemic (immunoglobulins: IgG, IgG1, IgG2a in plasma) and mucosal (IgA in fecal, lung, vaginal, saliva, and gut samples) humoral responses, as well as cell-mediated immune responses including T-cell proliferation and cytokines (interleukins: IL-4, IL-5; interferon: IFN- $\gamma$ ) were evaluated.
\end{abstract}

Results: CpG ODN, CT, and LT augmented antiHBs titers equally, and more so than did CTB or LTK63. CpG ODN acted synergistically with CT and LT, but not CTB or LTK63 to enhance anti-HBs titers. Nevertheless, CpG ODN induced a more Thl-like response for all combinations, compared with the same formulation without CpG. Strength of induced systemic and mucosal immune responses was better with IN delivery of a large volume. A small volume required multiple administrations and higher doses of antigen and adjuvant for equal results. This suggests that delivery of antigen to the lung and/or diges-tive system is superior to delivery to the nasal cavity.

Conclusions: Our results suggest that the synergy between CpG ODN and native toxins (CT, LT) may depend on their enzymatic activity and that the lack of synergy with nontoxic derivatives (LTB, LTK63) arises, since they do not have enzymatic activity. Because both CT and LT are too toxic for use in humans, it is possible that CpG ODN may be combined with bacterial toxin mutants that retain some enzymatic activity to optimize immune augmentation.

\section{Introduction}

The vast majority of infectious diseases are transmitted at the mucosal surfaces of the gas-

Address correspondence and reprint requests to: Dr. Heather L. Davis, Loeb Health Research Institute at the Ottawa Hospital, 725 Parkdale Avenue, Ottawa, KIY 4E9, Canada. Phone: 613-798-5555 ext. 7682; Fax: 613-761-5354; E-mail: hdavis@LRI.ca trointestinal, genitourinary, and respiratory tracts. To prevent infection at mucosal surfaces, an effective local immune response is required. However, most vaccines developed to date are delivered parenterally, for example by intramuscular (IM), subcutaneous, or intradermal injection, and this induces systemic, but little or no mucosal, immunity. Thus, there is a great 
need to develop vaccines that are effective when delivered by a mucosal route $(1,2)$. The principal inductive sites for the mucosal immune system, where antigens are encountered, endocytosed, and presented to B and T cells, are located in the: (i) gastrointestinal (GI) tract where gut-associated lymphoid tissue (GALT) includes the Peyer's patches (PP), appendix, mesenteric lymph nodes, and small solitary lymphoid nodules; (ii) respiratory tract with the bronchus-associated lymphoid tissue (BALT) and nasal-associated lymphoid tissue (NALT) or Waldeyer's ring (i.e., palatine, lingual, and nasopharyngeal tonsils); and (iii) genitourinary (GU) tract, which has the less well-characterized rectal-associated lymphoid tissue (RALT) of the large intestine $(1,2)$. Evidence for a "common mucosal immune system" (CMIS) is based on findings that antigen administration at one mucosal surface can result in protective immune responses at distant mucosal sites. This is thought to result from the distribution of surface immunoglobulin $\mathrm{A}^{+}$ $\left(\mathrm{IgA}^{+}\right) \mathrm{B}$ cells, $\mathrm{CD} 4^{+}$Thelper (Th) cells, and $\mathrm{CD8}^{+}$cytotoxic $\mathrm{T}$ lymphocytes (CTLs) cells from the inductive site to other mucosal effector sites $(1,2)$.

One of the major impediments to the development of mucosal vaccines has been the lack of effective, yet safe, mucosal adjuvants. Cholera toxin (CT) and the structurally related Escherichia coli heat-labile enterotoxin (LT) are potent mucosal adjuvants commonly used in animal models $(3,4)$. However, they are generally considered to be too toxic for use in humans. Two strategies have been proposed to circumvent the toxicity of the native toxins: (i) the use of the non-toxic B subunits that lack enzymatic activity $(5,6)$; or (ii) genetically detoxified mutants of CT or LT that have little or no remaining enzymatic activity. Such compounds, which have greatly reduced toxicity, retain some adjuvanticity in animal models (7-16). The efficacy of these new adjuvants has yet to be proven in humans.

A new class of adjuvant is CpG DNA, which may be administered in the form of synthetic oligodeoxynucleotides (ODN), containing immunostimulatory $\mathrm{CpG}$ motifs. Immunization of animals against a variety of antigens by parenteral delivery has shown that $\mathrm{CpG}$ DNA induces strong CTL and predominantly IgG2a antibodies, which are indicative of a Th1-type response (17-21). Recently, we and others showed CpG ODN to be a potent adju- vant for antigens delivered by intranasal (IN) inhalation (22-24). For example, we showed CpG ODN to be as potent as CT and that there were strong synergistic responses when the two adjuvants were used together (22). Although CT is too toxic for use in humans, these results suggested that $\mathrm{CpG}$ ODN might be combined with nontoxic derivatives of $\mathrm{CT}$, or of the closely related LT, to obtain better immune responses. Herein, we have examined this possibility using IN delivery of hepatitis B surface antigen (HBsAg) to mice as a model system. In addition to measurement of systemic and mucosal humoral responses, we also evaluated cell-mediated immune responses, including T-cell proliferation and cytokines. Finally we determined the effect of using small and large volumes, which would distribute antigen predominantly to the nasal cavity or the entire respiratory system, respectively, on immune responses after IN delivery.

\section{Materials and Methods}

\section{Immunization of Mice Against HBsAg}

Groups $(n=5-10)$ of female BALB/c mice (8-10 weeks, Charles River, Montreal, Quebec, Canada) were immunized by IN administration of 1 or $10 \mu \mathrm{g}$ HBsAg [plasma-derived hepatitis B virus (HBV) (S) protein, ad subtype, Genzyme Diagnostics, San Carlos, CA], alone or combined with 1, 10, or $50 \mu \mathrm{g}$ of CT (purified from Vibrio cholerae, Sigma, St. Louis, MO), LT (purified from Escherichia coli, Sigma), B subunit of CT (CTB; purified from Vibrio cholerae, Sigma), nontoxic derivative of LT (LTK63; mutant of LT bearing an Ser $\rightarrow$ Lys at position 63, generously provided by Dr. Rino Rappuoli, IRIS, Chiron S.P.A., Italy) and/or CpG ODN (5'-TCCATGACGTTCCTGACGTT-3') or the non-CpG control ODN (5'-TCCAGGACTTCT-CTCAGGTT3') (Hybridon Speciality Products, Milford, MA) previously described $(19,22)$. The antigen and adjuvant(s) were diluted to a total volume of $20 \mu \mathrm{l}$ (small volume) or $150 \mu \mathrm{l}$ (large volume) with $0.15 \mathrm{M} \mathrm{NaCl}$, and were administered by IN inhalation, as previously described (22). Both ODN had a nuclease-resistant phosphorothionate backbone (19). Some mice were boosted in an identical manner at 4 and 8 weeks after prime. Other mice that were immunized with $1 \mu \mathrm{g}$ HBsAg alone or with low-dose adjuvant $(1 \mu \mathrm{g})$, and that had no detectable immune re- 
sponse by 12 weeks were challenged by IM injection of $1 \mu \mathrm{g}$ HBsAg without adjuvant to determine if immune responses had been primed.

\section{Collection of Samples and Evaluation}

of Immune Responses

Plasma and fecal pellets were collected 1, 2, and 4 weeks post-immunization, and lung washes were carried out 4 weeks after final immunization, as previously described (22). Vaginal samples were collected by washing the vagina three times with $75 \mu \mathrm{l}(225 \mu \mathrm{l}$ total) of phosphate-buffered saline (PBS) containing $0.1 \mu \mathrm{g}$ sodium azide (Sigma). Saliva was obtained following i.p. injection with $100 \mu \mathrm{l}$ of 1 $\mathrm{mg} / \mathrm{ml}$ pilocarpine (Sigma) in PBS to induce saliva flow. Gut washes were obtained by removing the small intestine and passing $200 \mu \mathrm{l}$ PBS containing $0.1 \%$ sodium azide through each of three $10-\mathrm{cm}$ sections. All samples were stored at $-20^{\circ} \mathrm{C}$ until assayed by ELISA.

HBsAg-specific antibodies (anti-HBs) in the individual samples were detected and quantified by end-point dilution ELISA assay as described previously for IgG, IgG1, IgG2a (19) and IgA (22) isotypes. End-point dilution titers for IgG isotypes in plasma and IgA in mucosal samples were defined as the highest sample dilution that resulted in an absorbance value (OD 450) two times greater than that of non-immune plasma or mucosal sample, with a cut-off value of 0.05 . Anti-HBs titers for a group of animals were expressed as geometric mean titers \pm the standard error of the mean (GMT \pm SEM) of individual animal values, which were themselves the average of triplicate assays. Seroconversion was defined as a titer of $\geq 100$.

LYMPHOCYTE PROLIFERATION ASSAYS. Spleen cell suspensions were prepared and adjusted to a final concentration of $5 \times 10^{6}$ cells per $\mathrm{ml}$ in complete (RPMI 1640), which comprised RPMI 1640 tissue culture medium (Life Technologies, Grand Island, NY) supplemented with $2 \%$ normal mouse serum (Cedarlane Laboratories, Ontario, Canada), penicillin- streptomycin solution (final concentration of $1000 \mathrm{U} / \mathrm{ml}$ and 1 $\mathrm{mg} / \mathrm{ml}$, respectively; Sigma, Irvine, U.K.), and $5 \times 10^{-5}$ M $\beta$-mercaptoethanol (Sigma). The splenocyte suspension (100 $\mu \mathrm{l} /$ well $)$ was plated onto 96 -well U-bottomed tissue culture plates (100 $\mu \mathrm{l} / \mathrm{well})$, along with $100 \mu \mathrm{l}$ of each stimulant diluted to appropriate concentrations in complete RPMI 1640. The stimulant used was HBsAg (subtype ad, International En- zymes, Fallbrook, CA) at 5 and $2.5 \mu \mathrm{g} / \mathrm{ml}$. Concanavalin A (10 $\mu \mathrm{g} / \mathrm{ml}$; Sigma) was used as a positive control, and cells cultured with media alone were used as negative controls. Each splenocyte sample was plated in triplicate and the cells were incubated in a humidified $5 \%$ $\mathrm{CO}_{2}$ incubator for $96 \mathrm{hr}$ at $37^{\circ} \mathrm{C}$. The cells were then pulsed with $20 \mu \mathrm{Ci} / \mathrm{ml}(1 \mu \mathrm{Ci} /$ well $)$ tritiated thymidine (Amersham Pharmacia Biotech, Baie d'Urfé Quebec, Canada) and incubated for a further 16-18 hr. Next, the cells were harvested onto glass fiber filters using a cell harvester (Skatron Instruments, Norway) and the radioactivity incorporated into DNA was determined by liquid scintillation. Results were expressed as mean counts per minute (cpm) for three replicates.

CYTOKINE ASSAYs. Splenocytes from immunized animals were cultured with different stimuli, as described for lymphocyte proliferation assay. The cultures were incubated for 24 and $96 \mathrm{hr}$. At the end of the incubation period, the 96-well plates were centrifuged for $5 \mathrm{~min}$ at $1200 \mathrm{rpm}$ and culture supernatants harvested and stored at $-80^{\circ} \mathrm{C}$ until assayed. Commercially available assay kits (mouse IL-4 OptEIA, IL-5 OptEIA, and mouse IFN- $\gamma$ OptEIA; PharMingen, Mississauga Ontario, Canada) were used according to manufacturers' instructions to assay cytokine levels in culture supernatants taken at $24 \mathrm{hr}$ for IL-4 and IL-5 and at $96 \mathrm{hr}$ for IFN- $\gamma$.

\section{Statistical Analysis}

Data were analyzed using the GraphPAD InStat program (GraphPAD Software, San Diego, CA). The statistical significance of the difference between group means was calculated with nontransformed data for $\mathrm{T}$ cell proliferative responses or with transformed data $\left(\log _{10}\right)$ for ELISA titers by Student's 2-tailed $t$ test for two groups, or by one-factor analysis of variance (ANOVA), followed by Tukey's test for three or more groups. Differences were considered to be significant with $p<0.05$.

\section{Results}

Systemic Immune Responses (Large Volume)

We demonstrated previously that, with IN inhalation of HBsAg, CpG was equal to CT for induction of anti-HBs antibodies, and that they 
acted synergistically when used together (22). In that study, antigen and adjuvants were delivered in a $150 \mu$ l volume, which resulted in antigen delivery to the lungs and digestive tract, in addition to the nasal cavity. Although this is not an ideal model of an IN vaccine, we initially used the same large volume in the present study to determine whether a similar synergy could be obtained with CpG and other mucosal adjuvants, such as LT, CTB, or LTK63. IN delivery of HBsAg $(1 \mu \mathrm{g})$ without adjuvant did not induce detectable anti-HBs IgG antibodies in the plasma of any mice $(0 / 10)$. In contrast, high titers of anti-HBs IgG were induced in all mice when HBsAg was administered with $\mathrm{CpG}, \mathrm{CT}$, or LT (Figure 1). Although there was no statistical difference between anti-HBs IgG titers obtained with a low dose $(1 \mu \mathrm{g})$ of LT, CT, and CpG $(p=0.22)$, GMT titers were higher with CT and LT, than with $\mathrm{CpG}$, and there was greater intragroup variability associated with mice receiving $\mathrm{CpG}$ ODN. At a high dose $(10 \mu \mathrm{g}), \mathrm{CT}$ and LT gave higher titers than $\mathrm{CpG}$, however, $50 \%(5 / 10)$ of mice receiving this dose of LT died within 10 days. No detectable anti-HBs IgG was detected with a low dose $(1 \mu \mathrm{g})$ of CTB or LTK63, however, a high dose $(10 \mu \mathrm{g})$ of CTB gave low anti-HBs IgG endpoint ELISA titers and a high dose $(10 \mu \mathrm{g})$ of LTK63 gave strong levels of anti-HBs IgG, similar to a high dose $(10 \mu \mathrm{g})$ of CpG ( $p=0.97$; Figure 1). Thus, at low doses, CpG ODN as a mucosal adjuvant induced equivalent serum anti-HBs as the native toxins (CT, LT) and more than their nontoxic derivatives (CTB, LTK63).

When used together, CpG and either LT or CT (1 $\mu \mathrm{g}$ each) had synergistic effects, with anti-HBs titers being 5 to 10 times higher than with any of the three adjuvants alone (Figure 1). Indeed, CpG plus LT (1 $\mu$ g each) gave a better response than $10 \mu \mathrm{g}$ of either $\mathrm{CpG}$ or LT alone ( $p=0.007,0.015$, respectively) and the response with CpG plus CT ( $1 \mu \mathrm{g}$ each) was equal to that with $10 \mu \mathrm{g}$ CT alone $(p=$ $0.65)$. In contrast, there was no synergistic effect with LTK63 plus CpG (1 $\mu$ g each) for antiHBs IgG titers, which were equivalent to those with $1 \mu \mathrm{g} \mathrm{CpG}$ alone $(p=0.40)$. Surprisingly, CTB plus CpG ( $1 \mu \mathrm{g}$ each) gave lower anti-HBs titers than $1 \mu \mathrm{g} \mathrm{CpG}$ alone ( $p=0.007$; Figure 1$)$. Adjuvant effects with CpG ODN were due to the CpG motif, rather than a nonspecific effect of the ODN backbone, since mice immunized with $1 \mu \mathrm{g}$ of HBsAg plus $10 \mu \mathrm{g}$ of non-CpG

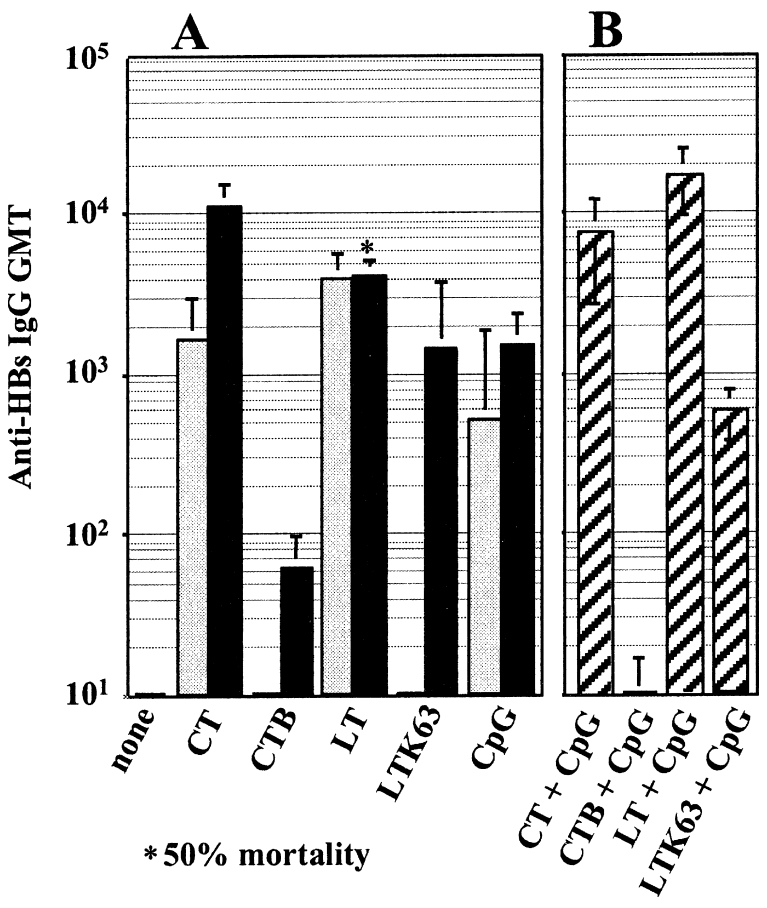

Fig. 1. HBsAg-specific antibody responses after intranasal (IN) immunization using large volume. BALB/c mice $(n=5-10)$ were immunized by a single IN inhalation of $150 \mu$ l solution containing $1 \mu \mathrm{g}$ HBsAg with no adjuvant (none), with $1 \mu \mathrm{g}$ (pale bars), or $10 \mu \mathrm{g}$ (black bars), of CT, CTB, LT, LTK63, or CpG ODN; or with CpG ODN combined with CT, CTB, LT, or LTK63 (1 $\mu \mathrm{g}$ each, striped bars). Each bar represents the group geometric mean titer (GMT) of the ELISA end-point dilution titer for HBsAg-specific antibodies [anti-HBs, total immunoglobulin G (IgG)] in plasma taken 4 weeks after immunization. Titers were defined as the highest plasma dilution resulting in an absorbance value two times that of non-immune plasma, with a cut-off value of 0.05 . HBsAg, purified hepatitis B surface antigen; CT, cholera toxin; CTB, subunit of cholera toxin; LT, Escherichia coli heat labile enterotoxin; LTK63, nontoxic derivative of Escherichia coli heat labile enterotoxin; CpG ODN, oligodeoxynucleotide containing cytosine-guanine phosphate linked dinucleotides.

ODN had no $(7 / 10)$ or very low (3/10) titers of anti-HBs IgG antibodies (data not shown). Therefore, a synergistic effect on levels of antiHBs IgG was seen when combining CpG ODN with native toxins (CT, LT), but not with their nontoxic derivatives (CTB, LTK63).

Antibody isotypes for the induced responses are shown in Figure 2. Antibodies were predominantly IgGl (Th2-like) with CT, CTB, and LT; and mixed IgG1/IgG2a (Th1/Th2) with LTK63. At a low dose of CpG (1 $\mu \mathrm{g})$, responses were mixed IgGl/IgG2a (Thl/Th2), but at a higher dose $(10 \mu \mathrm{g})$, they were more 


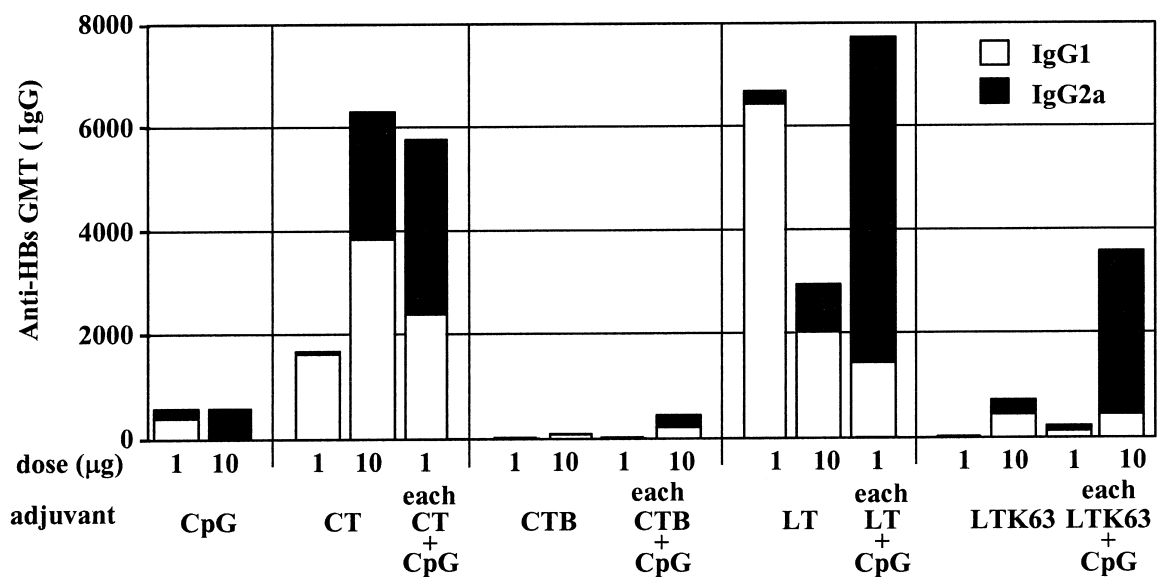

Fig. 2. HBsAg-specific immunoglobulin G (IgG) isotype responses after intranasal (IN) immunization using large volume. BALB/c mice $(n=$ 5-10) were immunized by a single IN inhalation of $1 \mu \mathrm{g}$ HBsAg with 1 or $10 \mu \mathrm{g}$ of CT, CTB, LT, LTK63, and/or CpG ODN as adjuvants in a volume of $150 \mu \mathrm{l}$. Each bar represents the group geometric mean of the ELISA end-point dilution titer for HBsAg-specific antibodies (anti-HBs geometric mean titers; GMT) of IgGl (white portion of bars)

Thl (IgG2a $>$ IgG1). With adjuvant combinations, responses were mixed Th1/Th2 with $\mathrm{CT} / \mathrm{CpG}$ or $\mathrm{CTB} / \mathrm{CpG}$, and more Thl with LT/CpG. At a low dose (1 $\mu$ g each), LTK63/ CpG responses were Th1/Th2, but at a higher dose (10 $\mu \mathrm{g}$ each), they were more Thl. Thus, coadministration of $\mathrm{CpG}$ with other adjuvants shifted responses towards a more Thl-like response, as indicated by a greater proportion of IgG2a antibodies. Thus, even with those adjuvants (CTB and LTK63) with which CpG ODN did not appear to act synergistically to augment levels of HBsAg-specific antibodies, the addition of $\mathrm{CpG}$ still promoted a more Thl-like response.

\section{Mucosal Immune Responses (Large Volume)}

When single adjuvants were used, only mice receiving LT (1 or $10 \mu \mathrm{g}$ ) or LTK63 (10 $\mu \mathrm{g})$ had detectable IgA in lung washes (GMT \pm SEM: $160 \pm 68,17 \pm 5,26 \pm 6$, respectively). Addition of CpG ODN caused some mice with $1 \mu \mathrm{g}$ CT (2/5), $10 \mu \mathrm{g}$ CTB (1/5), and $1 \mu \mathrm{g}$ LTK63 (1/5) to respond, although titers were low (results not shown). Only a few adjuvants on their own ( 1 or $10 \mu \mathrm{g}$ LT and $10 \mu \mathrm{g} \mathrm{CpG}$ ) induced IgA in the feces, and then only in some animals. No significant IgA was detected with CT, CTB, LTK63, or non-CpG control ODN. or IgG2a (black portion of bars) isotypes in plasma taken 4 weeks after immunization. Titers were defined as the highest plasma dilution resulting in an absorbance value two times that of nonimmune plasma, with a cut-off value of 0.05 . HBsAg, purified hepatitis B surface antigen; CT, cholera toxin; CTB, subunit of cholera toxin; LTK63, nontoxic derivative of Escherichia coli heat labile enterotoxin; CpG ODN, oligodeoxynucleotides containing cytosine-guanine phosphate-linked dinucleotides.

CpG and LT ( $1 \mu \mathrm{g}$ each) together resulted in IgA in the feces of a greater proportion of animals than $1 \mu \mathrm{g}$ of either adjuvant alone (CpG/LT 4/5, CpG 0/5, LT 2/5), suggesting an additive or synergistic effect. No such effects were evident with other combinations (results not shown).

\section{Systemic Humoral Immune Responses (Small Volume)}

To restrict delivery more to the nasal cavity, as would occur with nasal drops or nasal spray in a human, similar studies were carried out in mice using a volume of $20 \mu \mathrm{l}$, rather than 150 $\mu \mathrm{l}$. Although a single IN immunization with HBsAg ( $1 \mu \mathrm{g})$ combined with CT and/or CpG ( $1 \mu \mathrm{g}$ each) were effective in $150 \mu \mathrm{l}$ (Figure 1), they did not induce detectable antiHBs IgG antibodies in the plasma of any mice when given in $20 \mu \mathrm{l}$ (Table 1). When subsequently challenged by IM injection of HBsAg ( $1 \mu \mathrm{g})$ without adjuvant, some of these mice ( $3 / 5 \mathrm{CT}, 1 / 5 \mathrm{CpG}, 4 / 5 \mathrm{CT} / \mathrm{CpG}$ ) showed a memory response with a rapid rise in plasma IgG titers (to $10^{3}$ by week two), indicating that responses had been primed. No mice immunized with HBsAg alone in $20 \mu \mathrm{l}$ exhibited a primary or memory response (Table 1). 
Table 1. Effect of number of immunizations, antigen dose, and adjuvant dose on HBsAg-specific response ${ }^{a}$

\begin{tabular}{|c|c|c|c|c|c|c|c|}
\hline \multirow[b]{2}{*}{$\mathbf{N}^{\circ}$ Doses } & \multicolumn{3}{|c|}{ Adjuvant } & \multicolumn{4}{|c|}{ Number of Responders (of 5 immunized mice) } \\
\hline & HBsAg & CT & CpG & Post-prime & Post-boost 1 & Post-boost 2 & Post-challenge \\
\hline 1 & 1 & & & 0 & $\mathrm{~N} / \mathrm{D}^{\mathrm{b}}$ & N/D & 0 \\
\hline 1 & 1 & 1 & & 0 & N/D & N/D & 3 \\
\hline 1 & 1 & & 1 & 0 & N/D & N/D & 1 \\
\hline 1 & 1 & 1 & 1 & 0 & N/D & N/D & 4 \\
\hline 3 & 1 & & & 0 & 0 & 0 & 0 \\
\hline 3 & 1 & 1 & & 0 & 0 & 0 & 5 \\
\hline 3 & 1 & & 1 & 0 & 0 & 1 & 3 \\
\hline 3 & 1 & 1 & 1 & 0 & 0 & 2 & 5 \\
\hline 3 & 1 & 10 & & 1 & 2 & 4 & N/D \\
\hline 3 & 1 & & 10 & 0 & 3 & 5 & N/D \\
\hline 3 & 1 & & 50 & 0 & 1 & 3 & N/D \\
\hline 3 & 1 & 1 & 10 & 1 & 4 & 5 & N/D \\
\hline 3 & 10 & 1 & & 5 & 5 & 5 & N/D \\
\hline 3 & 10 & 10 & & 3 & 4 & 5 & N/D \\
\hline 3 & 10 & & 1 & 3 & 5 & 5 & N/D \\
\hline 3 & 10 & & 10 & 2 & 5 & 5 & N/D \\
\hline 3 & 10 & & 50 & 2 & 5 & 5 & N/D \\
\hline 3 & 10 & 1 & 1 & 5 & 5 & 5 & N/D \\
\hline 3 & 10 & 1 & 10 & 5 & 5 & 5 & N/D \\
\hline
\end{tabular}

${ }^{\mathrm{a} B A L B / c}$ mice were immunized by intranasal (IN) inhalation of 1 or $10 \mu \mathrm{g}$ of purified hepatitis B surface antigen (HBsAg) alone (none) or with 1, 10 or $50 \mu \mathrm{g}$ cholera toxin (CT) and/or CpG-containing oligodeoxynucleotides (CpG) in a total volume of $20 \mu \mathrm{l}$. Some mice were boosted in an identical manner at 4 and 8 weeks. Other mice that failed to develop anti-HBs, such as those immunized with $1 \mu \mathrm{g}$ HBsAg alone or with low dose adjuvant $(1 \mu \mathrm{g})$, were challenged by intramuscular (IM) injection of $1 \mu \mathrm{g}$ HBsAg without adjuvant, to determine if immune response had been primed. HBsAg-specific antibody titers were determined by ELISA in plasma taken 4 weeks after final immunization or 2 weeks after challenge. Titers were defined as the highest plasma dilution resulting in an absorbance value two times that of nonimmune plasma, with a cut-off value of 0.05 . Values shown represent the number of responding mice (titers $>100$ ).

${ }^{\mathrm{b}} \mathrm{N} / \mathrm{D}$, not determined

Even with triple immunizations using the small volume $(20 \mu \mathrm{l})$, no mice seroconverted when HBsAg ( $1 \mu \mathrm{g})$ was given without adjuvant or with CT, and only low numbers with CpG (1/5) or with the CpG/CT combination $(2 / 5)$. Nevertheless, upon challenge by IM injection of HBsAg ( $1 \mu \mathrm{g})$, most mice exhibited a memory response (5/5 CT, 3/5 CpG, and 5/5 CT/CpG; Table 1).

With the same small volume $(20 \mu \mathrm{l})$ and low dose of antigen ( $1 \mu \mathrm{g})$, increasing the dose of adjuvant (10 $\mathrm{g}$ CT; 10 or $50 \mu \mathrm{g} \mathrm{CpG)} \mathrm{re-}$ sulted in a higher proportion of responders in all groups. This was seen even with two doses, although for all formulations responses were better with three doses (Table 1).
When the dose of HBsAg was increased (10 $\mu \mathrm{g})$, all mice receiving it with $\mathrm{CT}$ or $\mathrm{CT} / \mathrm{CpG}$ seroconverted after a single immunization, as did most other mice after a single boost (Table 1). A single immunization using CT as adjuvant (1 or $10 \mu \mathrm{g}$ ) gave higher HBsAg-specific IgG titers in plasma than did 1, 10 or $50 \mu \mathrm{g}$ CpG ODN $(p<0.05)$. However, after boosting there was no difference between levels of HBsAg-specific IgG in plasma from mice with CT $(1$ or $10 \mu \mathrm{g})$ or CpG $(1,10$, or $50 \mu \mathrm{g} ; p>$ 0.05; Figure 3). Highest HBsAg-specific IgG titers were obtained when a combination of CpG and CT was used (Figure 3).

There were no differences in the IgG2a/ IgG 1 ratios after 1, 2, or 3 immunizations with 


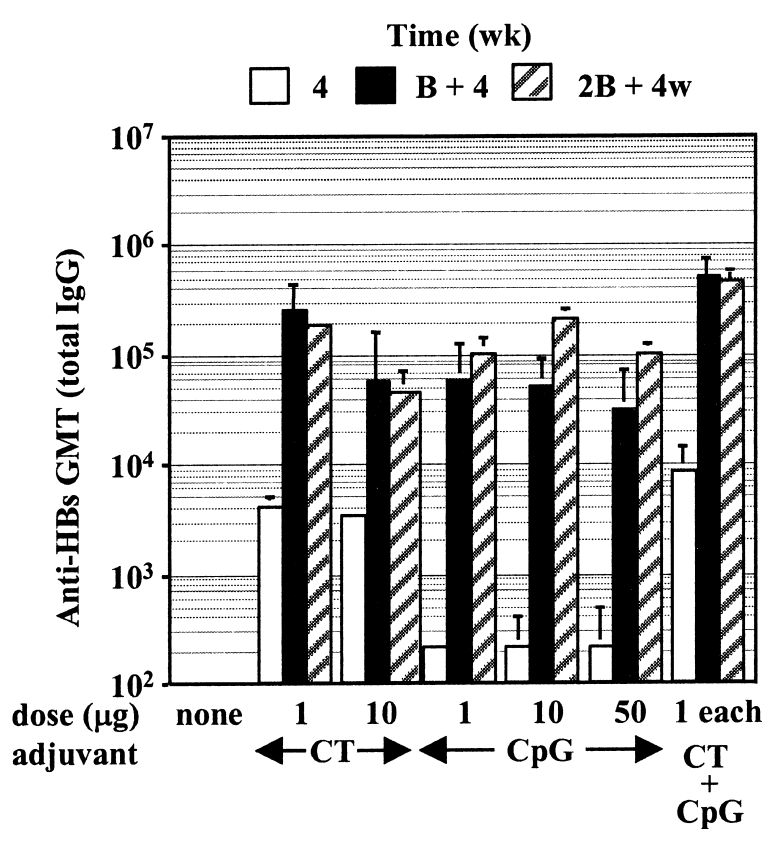

Fig. 3. HBsAg-specific antibody responses after intranasal (IN) immunization using small volume. BALB/c mice $(n=5-10)$ were immunized by IN inhalation of $10 \mu \mathrm{g}$ of HBsAg alone (none) or with 1, 10 or $50 \mu \mathrm{g}$ cholera toxin (CT) and/or CpG-containing oligodeoxynucleotides $(\mathrm{CpG})$ as adjuvants in a volume of $20 \mu \mathrm{l}$. Each bar represents the group mean $( \pm$ standard error of the mean; SEM) of the ELISA end-point dilution titer for HBsAg-specific antibodies (anti-HBs, total immunoglobulin G; IgG) in plasma taken 4 weeks after one (open bars), two (filled bars), or three (striped bars) immunizations. Titers were defined as the highest plasma dilution resulting in an absorbance value two times that of nonimmune plasma, with a cut-off value of 0.05 . HBsAg, purified hepatitis B surface antigen; GMT, geometric mean titers.

$10 \mu \mathrm{g}$ HBsAg given in a small volume $(20 \mu \mathrm{l})$ for mice within any particular group (results not shown), and therefore, only HBsAgspecific IgG isotypes after the third and final immunization are shown (Figure 4). Consistent with our earlier findings, when a large volume $(150 \mu \mathrm{l})$ was used, antibodies were predominantly IgGl (Th2-like) with $\mathrm{CT}$, and mixed IgG1/ IgG2a (Th1/Th2) with a low dose of CpG $(1 \mu \mathrm{g})$, but became more Thl (IgG2a $>$ IgGl) at higher doses of CpG. Responses were mixed Th1/Th2 with CT/CpG.

\section{Systemic Cellular Immune Responses (Small Volume)}

Using a $10 \mu \mathrm{g}$ antigen dose, HBsAg-specific $T$ cell proliferative responses were significantly

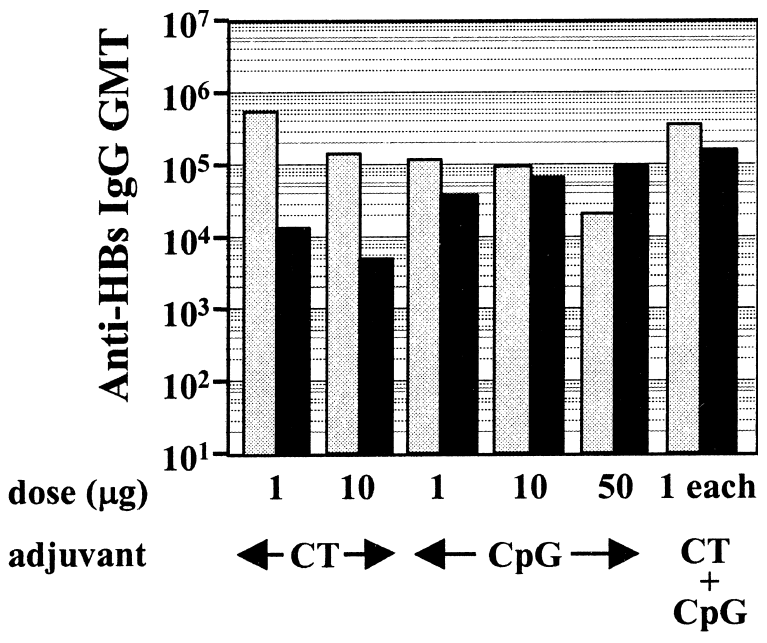

Fig. 4. HBsAg-specific immunoglobulin G (IgG) isotype responses after intranasal (IN) immunization using small volume. BALB/c mice $(n=$ $5-10)$ were immunized by IN inhalation of $10 \mu \mathrm{g}$ of HBsAg alone (none) or with 1, 10, or $50 \mu \mathrm{g}$ cholera toxin (CT) and/or CpG-containing oligodeoxynucleotides (CpG) as adjuvants in a volume of $20 \mu \mathrm{l}$. Mice were boosted at 4 and 8 weeks. Each bar represents the group geometric mean ( \pm standard error of the mean; SEM) of the ELISA endpoint dilution titer for HBsAg-specific antibodies (anti-HBs geometric mean titers; GMT) of IgGl (gray bars) or IgG2a (black bars) isotypes in plasma in plasma taken 4 weeks after the third and final immunization. Titers were defined as the highest plasma dilution resulting in an absorbance value two times that of non-immune plasma, with a cut-off value of 0.05. HBsAg, purified hepatitis B surface antigen.

enhanced $(p<0.05)$ by addition of CT and CpG (alone or in combination). Although the two adjuvants on their own gave equivalent levels of proliferation $(p=0.27)$, significantly stronger proliferative responses were induced when $\mathrm{CT}$ and $\mathrm{CpG}$ were used in combination $(p<0.015$; Figure 5). Responses were shown to be antigen-specific, since a much lower level of proliferation was obtained in cell cultures in the absence of HBsAg (data not shown). When culture supernatants from antigen-stimulated splenic $T$ cells from mice in these groups were examined by ELISA for IFN- $\gamma$, a Thl-type cytokine, CT, and CpG gave similar high levels, but the combination of CT and CpG gave extremely high levels (Figure 6). In contrast, IFN- $\gamma$ levels of unstimulated spleen cells were very low $(<100$ $\mathrm{pg} / \mathrm{ml}$ ) for all groups. Th2-type cytokines (IL-4 and IL-5) were not detectable in any groups (data not shown). 


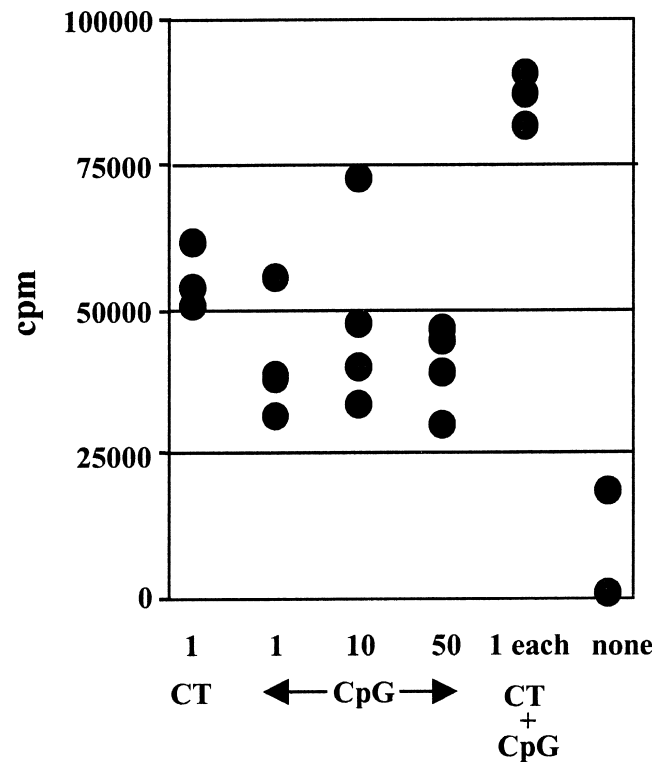

Fig. 5. HBsAg-specific $T$ cell proliferative responses after intranasal (IN) immunization (small volume). BALB/c mice $(n=5)$ were immunized by IN inhalation of $10 \mu \mathrm{g}$ of HBsAg alone (none) or with 1,10 , or $50 \mu \mathrm{g}$ cholera toxin (CT) and/or CpG-containing oligodeoxynucleotides $(\mathrm{CpG})$ as adjuvants in a volume of $20 \mu \mathrm{l}$. Mice were boosted at 4 and 8 weeks. Each circle represents the Ag-specific T cell proliferative responses expressed as counts per minute (cpm) for individual mice in splenocytes isolated 4 weeks after third and final immunization. HBsAg, purified hepatitis B surface antigen.

\section{Mucosal Immune Responses (Small Volume)}

Using a $10 \mu \mathrm{g}$ antigen dose, CT and CpG, alone or in combination, induced high levels of HBsAg-specific IgA in mucosal samples (Figure 7). With a $1 \mu \mathrm{g}$ adjuvant dose, levels of HBsAg-specific IgA using CpG were as high as those with CT in lung, gut, and saliva samples, but not as good in fecal or vaginal samples. However, IgA levels could be increased in a dose-dependent fashion in gut, fecal, saliva, and vaginal washes (but not in lung washes) with $\mathrm{CpG}$ as adjuvant (Figure 7), but not with CT (results not shown). Thus, by using higher doses of $\mathrm{CpG}$, it was possible to attain in all mucosal samples equivalent (lung, gut, fecal) or better (saliva, vaginal) levels of IgA than could be obtained with CT. A combination of CT and CpG ( $1 \mu \mathrm{g}$ each) gave better responses than either adjuvant alone in lung, saliva, and fecal samples, but not in gut or vaginal samples.

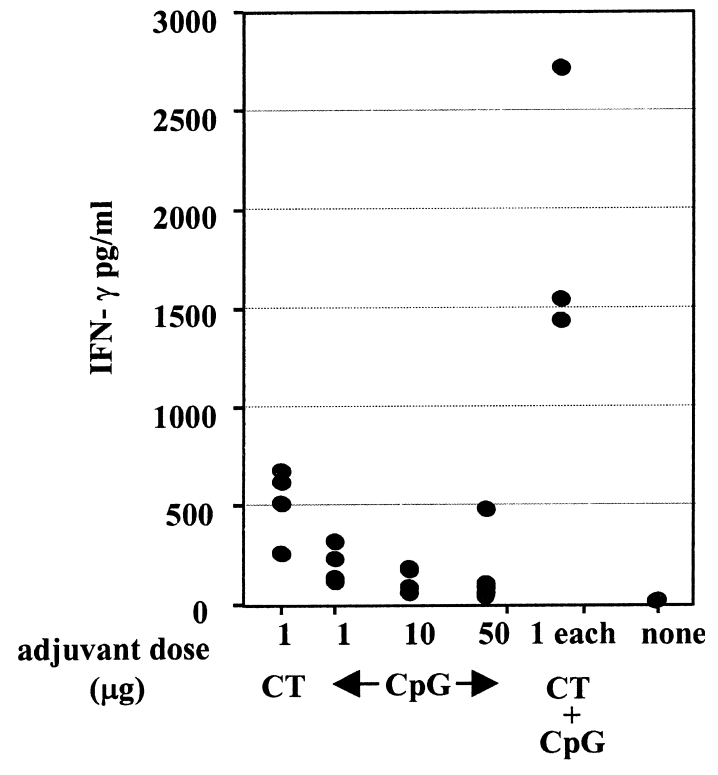

Figure 6. Interferon- $\gamma($ IFN- $\gamma)$ secretion from splenocytes isolated from BALB/c after intranasal (IN) immunization (small volume). BALB/c mice $(n=5)$ were immunized by IN inhalation of $10 \mu \mathrm{g}$ of HBsAg alone (none) or with 1, 10, or $50 \mu \mathrm{g}$ cholera toxin (CT) and/or CpGcontaining oligodeoxynucleotides (CpG) as adjuvants in a volume of $20 \mu \mathrm{l}$. Mice were boosted at 4 and 8 weeks. Culture supernatants taken at 96 hr from Ag-stimulated splenic T cells from mice in these groups were examined by ELISA for IFN- $\gamma$. Each circle represents the level of IFN- $\gamma$ in culture supernatant from an individual mouse expressed in pg/ml. HBsAg, purified hepatitis B surface antigen.

\section{Discussion}

Intranasal immunization is desirable for mass vaccination, since it is fast and easy to administer, requires minimal training of personnel, and carries no risk of needle stick injury or cross contamination. CT and LT have been widely used as adjuvants for IN immunization of animals $(3,4)$, but they are too toxic for use in humans. We previously showed with IN delivery of HBsAg to mice, CpG ODN was nontoxic, but equal to $C T$, for augmenting plasma IgG and lung IgA (22). Here we show that CpG also enhances IgA production in gut, fecal, saliva, and vaginal samples. These IgA responses at the mucosal surfaces of the respiratory, gastrointestinal, and genitourinary tracts indicate that CpG ODN stimulates the CMIS. We also previously reported a strong synergistic effect when CpG ODN was used together with CT (22), and now show this also to be true with LT. This is not surprising considering the structural and 


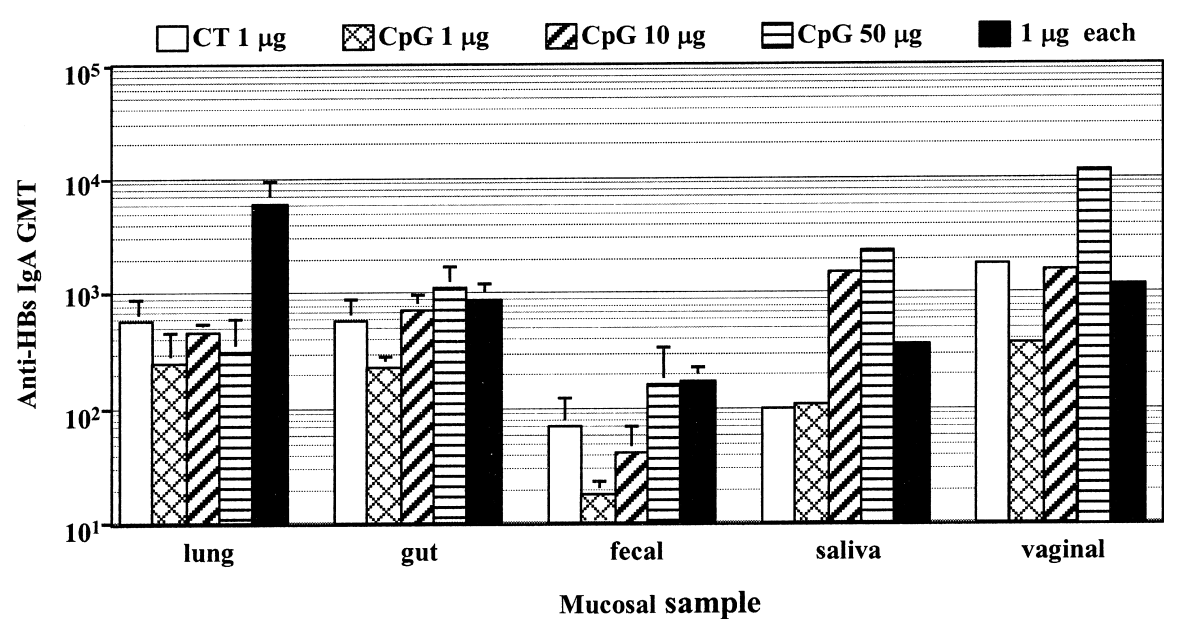

Fig. 7. HBsAg-specific immunoglobulin A (IgA) responses after intranasal (IN) immunization (small volume). BALB/c mice $(n=5-10)$ were immunized by IN inhalation of $10 \mu \mathrm{g}$ of HBsAg with 1, 10, or $50 \mu \mathrm{g}$ cholera toxin (CT) and/or CpG-containing oligodeoxynucleotides (CpG) as adjuvants in a volume of $20 \mu \mathrm{l}$. Mice were boosted at 4 and 8 weeks. Each bar represents the group geometric mean of the ELISA end-point dilution

functional similarities between CT and LT $(3,4)$, which have $80 \%$ homology in sequence. Both CT and LT comprise two subunits, a monomeric A subunit with enzymatic activity and a pentameric ring-shaped $B$ subunit that binds ganglioside GMl at the surface of eukaryotic cells and enables insertion of the A subunit into the cytosol, where it ADP-ribosylates a GTP-binding regulatory protein associated with adenylate cyclase (4). Despite these similarities, we found LT to be more potent than CT, especially for induction of mucosal IgA; but, it was also more toxic.

No overt toxicity has been detected with CpG, as measured by morbidity (piloerection, loss of weight, arched back, withdrawal) and mortality, even at very high doses (up to 500 $\mu \mathrm{g})$ (22). Although the synergistic effect of CpG ODN allows lower doses of CT and LT to be used in mice, the native forms of these toxins are unlikely to be used in humans because of their toxicity, even at very low doses. A mouse can tolerate doses of up to $10 \mu \mathrm{g}$ of native toxin; whereas, an equivalent dose will cause severe diarrhea in humans $(25,26)$.

When we tested CpG ODN with nontoxic derivatives of CT and LT, namely CTB and LTK63, no synergistic effect on titers of IgG or IgA were noted. Indeed, when CTB was coadministered with $\mathrm{CpG}$, it appeared to inhibit the titer (GMT) for HBsAg-specific IgA antibodies (anti-HBs IgA GMT) in lung and gut washes, feces, saliva, and vaginal washes collected 4 weeks after final immunization. Titers were defined as the highest sample dilution resulting in an absorbance value two times that of non-immune sample, with a cut-off value of 0.05 . HBsAg, purified hepatitis B surface antigen.

adjuvant effect of $\mathrm{CpG}$, perhaps due to an inhibition of CpG and/or antigen uptake. Nevertheless, when CpG was coadministered with either CTB or LTK63, the Th1-bias of the response (IgG2a > IgG1) was more pronounced than without CpG. These results indicate that the synergy between CpG ODN and CT or LT may depend on their enzymatic activity. Indeed, it has been suggested that the adjuvant activity of CT or LT is dependent on this enzymatic activity, although results are conflicting. Several investigators have reported that recombinant CTB fails to stimulate mucosal responses against admixed antigens $(3,14,15)$ or is significantly weaker than CT $(27,28)$; whereas, others report strong adjuvant activity of LTB or CTB $(6,29)$. Here we found CTB to have weak adjuvant activity, but this might be due to contamination with residual holotoxin, since CTB purified from native CT was used $(30,31)$. Likewise, with genetically detoxified mutants of CT and LT (7-16), the importance of residual enzymatic activity is not clear. In some studies, mutants lacking enzymatic activity fail to generate antibody responses against coadministered antigen (3); whereas, in other studies they appeared to retain the adjuvant properties of the native toxin $(10,13,32,33)$. We found the LTK63 mutant that lacks enzymatic activity $(7,8,10)$ to be moderately effective as a mucosal adjuvant, but only at high doses. It has been suggested that 
better adjuvanticity may be obtained with mutants that retain some enzymatic activity, e.g. LTR72 or CTS $106(12,33)$, and it is also possible that better synergy with CpG ODN would be realized with such partially active toxins.

Of all adjuvants tested on their own, CpG ODN gave the strongest Thl-type responses. Furthermore, in all combinations containing CpG ODN, responses were pushed in a Thl direction, compared with those with the same formulation minus the CpG ODN. Thl-like responses are desirable because of the association with enhanced levels of neutralizing IgG2a antibodies and CTL activity. Furthermore, ThI responses may be particularly desirable in the lungs, since allergic asthma is generally associated with Th2-like responses $(34,35)$. Interestingly, CpG recently was shown in mice to prevent allergen-induced asthmatic responses, including airway eosinophilia, Th2 cytokine induction, IgE production, and bronchial hyperactivity, and to redirect the immune response towards a more Thl-like profile $(36,37)$.

Regardless of the adjuvant used, a large volume was better than a small volume for immunization of mice by IN inhalation. To obtain equivalent results with a small volume, it was necessary to use higher doses of antigen and adjuvant, and more administrations. This suggests that certain mucosal inductive sites may be more effective than others. With IN inhalation of a large volume, antigen delivery would be to lymphoid tissue in both the upper and lower respiratory tracts (i.e., nasal associated lymphoid tissue, bronchial associated lymphoid tissue [NALT, BALT]), and most certainly some would be swallowed, so CpG might also be delivered to lymphoid tissue in the GI tract (e.g., PP). In contrast, with IN inhalation of a small volume, the upper respiratory tract (i.e., NALT) would be the primary site of antigen delivery. Our results in mice, where stronger immune responses were obtained with large volumes, suggest that delivery of antigen to the lung and/or digestive system is superior to delivery to the nasal cavity. Indeed, we showed CpG on its own to be a highly effective adjuvant, even at low doses, with oral delivery (McCluskie and Davis, unpublished observations). The role of the lung per se as an inductive site remains to be determined. Prior to application in humans, it is desirable to confirm the mucosal adjuvant effects of CpG ODN and the relative importance of different mucosal inductive sites in nonhuman primates. This may indicate whether it would be better to use an oral vaccine, an inhaled neb- ulized vaccine, or a purely intranasal one, such as a nasal spray or nose drops.

\section{Acknowledgments}

We are grateful to Brian Avery, Amanda Boyd, Lacrimioara Comanita, George Condrut, Karen Wong, and Lu Zhang for excellent technical assistance. This research was supported by the MRC (Canada) and Coley Pharmaceutical Group, Inc., (Wellesley, MA). HLD is a recipient of a Career Scientist Award from the Ontario Ministry of Health.

\section{References}

1. McGhee JR, Mestecky J, Dertzbaugh MT, Eldridge JH, Hirasawa M, Kiyono H. (1992) The mucosal immune system: from fundamental concepts to vaccine development. Vaccine 10: 75-88.

2. Nugent J, Po AL, Scott EM. (1998) Design and delivery of non-parenteral vaccines. J. Clin. Pharm. Ther. 23: 257-285.

3. Lycke N, Tsuji T, Holmgren J. (1992) The adjuvant effect of Vibrio cholerae and Escherichia coli heat-labile enterotoxins is linked to their ADPribosyltransferase activity. Eur. J. Immunol. 22: 2277-2281.

4. Spangler BD. (1992) Structure and function of cholera toxin and the related Escherichia coli heatlabile enterotoxin. Microbiol. Rev. 56: 622-647.

5. Holmgren J, Lycke N, Czerkinsky C. (1992) Cholera toxin and cholera B subunit as oralmucosal adjuvant and antigen vector systems. Vaccine 11: 1179-1184.

6. Verweij WR, de Haan L, Holtrop M, et al. (1998) Mucosal immunoadjuvant activity of recombinant Escherichia coli heat-labile enterotoxin and its B subunit: induction of systemic IgG and secretory IgA responses in mice by intranasal immunization with influenza virus surface antigen. Vaccine 16: 2069-2076.

7. Rappuoli R, Douce G, Dougan G, Pizza M. (1995) Genetic detoxification of bacterial toxins: a new approach to vaccine development. Int. Arch. Allergy App. Immunol. 108: 327-333.

8. Fontana MR, Manetti R, Giannelli V, et al. (1995) Construction of nontoxic derivatives of cholera toxin and characterization of the immunological response against the A subunit. Infect. Immun. 63: 2356-2360.

9. Chong C, Friberg M, Clements JD. (1998) LT (R192G), a non-toxic mutant of the heatlabile enterotoxin of Escherichia coli, elicits enhanced humoral and cellular immune responses associated with protection against lethal oral challenge with Salmonella spp. Vaccine 16: 732- 740. 
10. Di Tommaso A, Saletti G, Pizza M, et al. (1996) Induction of antigen-specific antibodies in vaginal secretions by using a nontoxic mutant of heat-labile enterotoxin as a mucosal adjuvant. Infect. Immun. 64: 974-979.

11. Douce G., Giuliani MM, Giannelli V, Pizza MG, Rappuoli R, Dougan G. (1998) Mucosal immunogenicity of genetically detoxified derivatives of heat labile toxin from Escherichia coli. Vaccine 16: 1065-1073.

12. Giuliani MM, Del Giudice G, Giannelli V, et al. (1998) Mucosal adjuvanticity and immunogenicity of LTR72, a novel mutant of Escherichia coli heat-labile enterotoxin with partial knockout of ADP- ribosyltransferase activity. J. Exp. Med. 187: 1123-1132.

13. Komase K, Tamura S, Matsuo K, et al. (1998) Mutants of Escherichia coli heat-labile enterotoxin as an adjuvant for nasal influenza vaccine. $\mathrm{Vac}$ cine 16: 248-254.

14. Yamamoto S, Kiyono $H$, Yamamoto $M$, et al. (1997) A nontoxic mutant of cholera toxin elicits Th2-type responses for enhanced mucosal immunity. Proc. Natl. Acad. Sci. U.S.A. 94: 5267- 5272.

15. Yamamoto S, Takeda Y, Yamamoto $M$, et al. (1997) Mutants in the ADP-ribosyltransferase cleft of cholera toxin lack diarrheagenicity but retain adjuvanticity. J. Exp. Med. 185: 1203-1210.

16. Yamamoto $M$, Briles DE, Yamamoto $S$, Ohmura M, Kiyono H, McGhee JR. (1998) A nontoxic adjuvant for mucosal immunity to pneumococcal surface protein A. J. Immunol. 161: 4115-4121.

17. Roman M, Martin-Orozco E, Goodman JS, et al. (1997) Immunostimulatory DNA sequences function as T helper-1-promoting adjuvants. Nat. Med. 3: 849-854.

18. Chu RS, Targoni OS, Krieg AM, Lehmann PV, Harding CV. (1997) CpG oligodeoxynucleotides act as adjuvants that switch on T helper 1 (Thl) immunity. J. Exp. Med. 186: 1623-1631.

19. Davis HL, Weeranta R, Waldschmidt TJ, Tygrett L, Schorr J, Krieg AM. (1998) CpG DNA is a potent enhancer of specific immunity in mice immunized with recombinant Hepatitis B surface antigen. J. Immunol. 160: 870-876.

20. Lipford GB, Sparwasser T, Bauer M, et al. (1997) Immunostimulatory DNA: sequence-dependent production of potentially harmful or useful cytokines. Eur. J. Immunol. 27: 3420-3426.

21. Weiner GJ, Liu HM, Wooldridge JE, Dahle CE, Krieg AM. (1997) Immunostimulatory oligodeoxynucleotides containing the CpG motif are effective as immune adjuvants in tumor antigen immunization. Proc. Natl. Acad. Sci. U.S.A. 94: 10833- 10837.

22. McCluskie MJ, Davis HL. (1998) CpG DNA is a potent enhancer of systemic and mucosal immune responses against hepatitis B surface antigen with intranasal administration to mice. J. Immunol. 161: 4463-4466.
23. Horner AA, Ronaghy A, Cheng PM, et al. (1998) Immunostimulatory DNA is a potent mucosal adjuvant. Cell. Immunol. 190: 77-82.

24. Moldoveanu Z, Love-Homan L, Huang WQ, Krieg AM. (1998) CpG DNA, a novel immune enhancer for systemic and mucosal immunization with influenza virus. Vaccine 16: 1216- 1224.

25. Michetti P, Kreiss C, Kotloff K, et al. (1997) Oral immunization of $H$. pylori infected adults with recombinant urease and LT adjuvant. Gastroenterol. 112: A1042.

26. Levine MM, Kaper JB, Black RE, Clements ML. (1983) New knowledge on pathogenesis of bacterial enteric infections as applied to vaccine development. Microbiol. Rev. 47: 510- 550.

27. Wu HY, Russell MW. (1998) Induction of mucosal and systemic immune responses by intrana-sal immunization using recombinant cholera toxin B subunit as an adjuvant. Vaccine 16: 286- 292.

28. Blanchard TG, Lycke N, Czinn SJ, Nedrud JG. (1998) Recombinant cholera toxin B subunit is not an effective mucosal adjuvant for oral immunization of mice against Helicobacter felis. Immunology 94: 22-27.

29. Tochikubo K, Isaka M, Yasuda Y, et al. (1998) Recombinant cholera toxin B subunit acts as an adjuvant for the mucosal and systemic responses of mice to mucosally co-administered bovine serum albumin. Vaccine 16: 150- 155.

30. Tamura S, Yamanaka A, Shimohara M, et al. (1994) Synergistic action of cholera toxin B subunit (and Escherichia coli heat-labile toxin B subunit) and a trace amount of cholera whole toxin as an adjuvant for nasal influenza vaccine. Vaccine 12: 419-426.

31. Tamura S, Asanuma H, Tomita T, et al. (1994) Escherichia coli heat-labile enterotoxin B subunits supplemented with a trace amount of the holotoxin as an adjuvant for nasal influenza vaccine. Vaccine 12: 1083-1089.

32. Douce G, Turcotte C, Cropley I, et al. (1995) Mutants of Escherichia coli heat-labile toxin lacking ADP-ribosyltransferase activity act as nontoxic, mucosal adjuvants. Proc. Natl. Acad. Sci. U.S.A. 92: 1644-1648.

33. Douce G, Fontana M, Pizza M, Rappuoli R, Dougan G. (1997) Intranasal immunogenicity and adjuvanticity of site-directed mutant derivatives of cholera toxin. Infect. Immun. 65: 2821-2828.

34. Kay AB. (1996) TH2-type cytokines in asthma. Ann. NY Acad. Sci. 796: 1-8.

35. Hogg JC. (1997) The pathology of asthma. APMIS 105: 735-745.

36. Kline JN, Waldschmidt TJ, Businga TR, (1998) Modulation of airway inflammation by $\mathrm{CpG}$ oligodeoxynucleotides in a murine model of asthma. J. Immunol. 160: 2555-2559.

37. Broide D, Schwarze J, Tighe H, et al. (1998) Immunostimulatory DNA sequences inhibit IL-5, eosinophilic inflammation, and airway hyperresponsiveness in mice. J. Immunol. 161: 7054- 7062. 